\title{
Recalcitrant carbohydrates after enzymatic hydrolysis of pretreated lignocellulosic biomass
}

\author{
María Ángeles Bermúdez Alcántara', Justyna Dobruchowska², Parastoo Azadi², Bruno Díez García', \\ Fernando P. Molina-Heredia ${ }^{3}$ and Francisco Manuel Reyes-Sosa ${ }^{1 *}$
}

\begin{abstract}
Background: To reduce the cost of the enzymes for the hydrolysis of lignocellulosic biomass, two main strategies have been followed: one, the reduction of enzyme dosing by the use of more efficient and stable enzymatic cocktails; another, to include accessory enzymes in the cocktails to increase yields by reducing the recalcitrant carbohydrate fraction remaining at the end of the process. To guide this second strategy, we have explored the chemical bond composition of different fractions of recalcitrant carbohydrates after enzymatic hydrolysis.

Results: Two lignocellulosic feedstocks of relevance for the biofuels industry have been analyzed, corn stover and sugarcane straw. On comparing the composition of chemical bonds of the starting pretreated material with samples after standard and forced hydrolysis (with enzyme overdosing), we obtained similar sugar and chemical bond composition.

Conclusions: This suggests that the current enzymatic cocktails bear the set of enzymes needed to hydrolyze these feedstocks. From our point of view, the results show the need for a parallel fine-tuning of the enzymatic cocktails with the pretreatment process to maximize sugar release yield.
\end{abstract}

Keywords: Enzymatic hydrolysis, Lignocellulosic biomass, Recalcitrant carbohydrates, Bioethanol

\section{Background}

The interest of biochemical deconstruction of lignocellulosic biomass into sugars for the production of ethanol fuel or chemicals has been increasing in the last decades due to oil prices volatility, together with the lower carbon emissions profile of the biochemical route with direct benefit in the mitigation of global warming and climate change. Lignocellulosic materials are a renewable and abundant source of carbon for the production of fuels and chemicals. They can be obtained from low-cost resources like agricultural and forest residues, municipal solid waste, waste paper and energy crops. These materials contain polymeric sugars that can be hydrolyzed and

\footnotetext{
*Correspondence: francisco.reyes@abengoa.com

1 Department of Biotechnology, Abengoa Research, Campus Palmas

Altas, C/Energía Solar no 1, 41014 Seville, Spain

Full list of author information is available at the end of the article
}

subsequently fermented to ethanol by microorganisms [1].

Lignocellulosic material is composed mainly of three polymers: cellulose, hemicellulose and lignin, which are associated in a complex matrix with different composition depending on the type, species and source of the biomass [2]. The presence of these complex interlinked polymers presents a physical barrier to protect cellulose from degradation. This compact and rigid structure is the cause of biomass recalcitrance to hydrolysis and deconstruction. The factors that contribute to biomass recalcitrance include: crystallinity and degree of polymerization of cellulose; accessible surface area (or porosity); protection of cellulose by lignin; cellulose sheathing by hemicellulose; and fiber strength [3, 4].

While the enzymatic breakdown of lignocellulosic biomass is a complicated process, involving many activities which work in tandem to decompose a heterogeneous 
and naturally recalcitrant substrate, the understanding of both known and yet-to be discovered enzymes and activities has increased significantly in recent years [5].

To increase the yield of enzymatic hydrolysis at the industrial scale, a physico-chemical pretreatment of the biomass is required, whose main objective is altering the chemical composition and physical structures of biomass to remove the recalcitrant barriers and enhance the enzymatic digestibility of cellulose, to allow the exposed complex carbohydrates such as cellulose and hemicelluloses to be readily hydrolyzed to fermentable sugars.

There are many comprehensive reviews where different pretreatment technologies based on physical, chemical and biological methods have been described $[2,3,6]$. Basically, an effective pretreatment method should be cheap (both in capital and operating costs), effective on a wide range of lignocellulosic materials, require minimum preparation/handling steps prior to pretreatment, ensure recovery of all of the lignocellulosic components in a useable form, and provide a cellulosic stream that can be efficiently hydrolyzed with low concentrations of enzymes [2].

Due to the interplay of the efficiency of the pretreatment with the enzymatic hydrolysis yields, it is often difficult to ascertain which of the two processes limits the overall yield. One possible limitation could be that the enzyme mixture would lack specific activities to unlock key chemical bonds in the polymers, thus limiting the overall sugar yield. An alternative hypothesis would be that the cocktail would be adequately formulated, but a portion of the biomass could be inefficiently pretreated, provoking a limitation on enzymatic hydrolysis yield. To test these hypothesis, in the present study we characterized two different lignocellulosic materials, corn stover and sugarcane straw, both pretreated by diluted acid/ steam explosion, following hydrolysis under low and high enzyme loading. After the hydrolysis, the chemical composition of the recalcitrant materials was analyzed to unveil the composition of these recalcitrant materials using several approaches such as solid-state nuclear magnetic resonance (NMR) spectroscopy, glycosyl composition determined by using gas chromatography/ mass spectrometry (GC/MS) of the per-O-trimethylsilyl (TMS) derivatives, and per-O-methylation linkage analysis of glycosyl residues to have a global view of the chemical linkages present in the material as compared to the starting pretreated material. No different chemical bond composition was found when starting and recalcitrant materials were compared. This supports the hypothesis that pretreatment rather than enzymatic cocktail reformulation represents the main bottleneck in sugar release yields.

\section{Methods}

\section{Corn stover and sugarcane straw}

Pretreated corn stover and sugarcane straw (from now on, PCS and PSCS, respectively) were obtained from Abengoa Bioenergy Biomass Pilot Plant in York, Nebraska, USA. A 1-inch hammer mill screen was used to grind the material. Pretreatment of the milled material was performed by steam explosion using $2.3 \%(\mathrm{w} / \mathrm{v})$ sulfuric acid for spray impregnation of the biomass in a continuous digester, previous to pressurizing with steam at 150 psig for $2 \mathrm{~min}$; a final dry matter content of 37.4 and $43.4 \%$ was achieved, respectively, for PCS and PSCS. The compositional analysis of the biomass was according to the standard biomass analytical procedures by NREL [7].

\section{Enzymatic hydrolysis}

Hydrolysis of the pretreated biomass (20 g) was performed in $100 \mathrm{~mL}$ borosilicate glass bottles with airtight screw caps. Water was added to adjust the solid loading to $20 \%$ of water insoluble solids (WIS; based on substrate). The $\mathrm{pH}$ was initially adjusted to 5.5 by addition of aqueous $\mathrm{NH}_{4} \mathrm{OH}$. The enzyme loading was 10 and $100 \mathrm{mg}$ protein per gram glucan of $\mathrm{C} 1$ enzyme preparation (supplied by Abengoa). The hydrolysis was incubated at $50{ }^{\circ} \mathrm{C}$ with orbital shaking at $150 \mathrm{rpm}$ for $72 \mathrm{~h}$. Samples were taken at $t=0$ and $t=72 \mathrm{~h}$ of hydrolysis and were processed for analysis according to Kristensen et al. [8] due to the higher density of the hydrolysate at $20 \%$ of WIS. The analytes were quantified in weight/weight (g/ $\mathrm{kg})$.

\section{Carbohydrate analysis}

After enzymatic hydrolysis, samples were filtered and analyzed by high-performance liquid chromatography (HPLC) using an Aminex HPX-87H $300 \mathrm{~mm} \times 7.8 \mathrm{~mm}$ column with $9 \mu \mathrm{m}$ particle size (Bio-Rad, California, USA). The analyses were performed at $60{ }^{\circ} \mathrm{C}$ under isocratic conditions with $5 \mathrm{mM} \mathrm{H}_{2} \mathrm{SO}_{4}$ as mobile phase at a flow rate of $0.6 \mathrm{~mL} / \mathrm{min}$ with $20 \mu \mathrm{L}$ injection volume. Carbohydrates (glucose, xylose and arabinose) were analyzed using a refractive index detector.

\section{Sample preparation}

After hydrolysis, the enzymes were inactivated by boiling for $15 \mathrm{~min}$ at $100{ }^{\circ} \mathrm{C}$. The samples were centrifuged $\left(16,000 \times g, 30 \mathrm{~min}, 4^{\circ} \mathrm{C}\right)$ and the pellets washed with Milli-Q water at $50^{\circ} \mathrm{C}$ for $1 \mathrm{~h}$, repeating the washing until no soluble sugars were detected by HPLC in the supernatants. Then, recalcitrant materials were freeze dried. Dry samples were milled in a hammer mill, passed through a $250 \mu \mathrm{m}$ sieve and their humidity content determined 
using a Shimadzu (Kyoto, Japan) heating balance. All the component contents are expressed on dry weight basis as average with a standard deviation of duplicate determinations for each sample.

\section{Glycosyl composition by GC/MS of TMS derivatives of methyl glycosides}

Glycosyl composition analysis was performed by combined GC/MS of the per-O-trimethylsilyl derivatives of the monosaccharide methyl glycosides produced from the sample by acidic methanolysis. $400 \mu \mathrm{g}$ samples were placed in a screw-cap tube with $20 \mu \mathrm{g}$ of inositol as internal standard and hydrolyzed with $2 \mathrm{M}$ trifluoroacetic acid (TFA) at $120{ }^{\circ} \mathrm{C}$ for $1 \mathrm{~h}$. The hydrolysis products were dried by adding iso-propanol and methanol to remove TFA. Methyl glycosides were then prepared from the freeze-dried sample following the mild acid treatment by methanolysis in $1 \mathrm{M} \mathrm{HCl}$ in methanol at $80{ }^{\circ} \mathrm{C}(16 \mathrm{~h})$, followed by re- $\mathrm{N}$-acetylation with pyridine and acetic anhydride in methanol (for detection of amino sugars). The sample was then $O$-trimethylsilylated by treatment with Tri-Sil at $80{ }^{\circ} \mathrm{C}(0.5 \mathrm{~h})$. These procedures were carried out as previously described by Merkle and Poppe [9]. GC/MS analysis of the TMS methyl glycosides was performed on an Agilent 7890A GC interfaced to a 5975C MSD, using a Supelco EC-1 fused silica capillary column $(30 \mathrm{~m} \times 0.25 \mathrm{~mm}$ ID).

\section{Per-O-methylation and linkage analysis of neutral sugars} For glycosyl linkage analysis, the sample was permethylated, depolymerized, reduced, and acetylated. The resulting partially methylated alditol acetates (PMAAs) were analyzed by GC/MS as described by York et al. [10]. Initially, dry samples were suspended in about $300 \mu \mathrm{L}$ of dimethyl sulfoxide and placed on a magnetic stirrer for 1 week. The samples were then permethylated by the method of Ciucanu and Kerek (treatment with sodium hydroxide and methyl iodide in dry DMSO) [11]. Each sample was incubated with $\mathrm{NaOH}$ for 15 min, then methyl iodide was added and left for $45 \mathrm{~min}$. The base was then added for $10 \mathrm{~min}$ and finally more methyl iodide was added for $40 \mathrm{~min}$. This addition of more methyl iodide and $\mathrm{NaOH}$ base was to ensure complete methylation of the polymer. Following the sample workup, the permethylated material (PMAA) was hydrolyzed using $2 \mathrm{M}$ TFA ( $2 \mathrm{~h}$ in sealed tube at $121^{\circ} \mathrm{C}$ ), reduced with $\mathrm{NaBD}_{4}$, and acetylated using acetic anhydride/TFA. The resulting PMAAs were analyzed on a Hewlett Packard 7890A GC interfaced to a 5975C MSD mass selective detector, electron impact ionization mode (EI-MS); separation was performed on a $30 \mathrm{~m}$ Supelco 2380 bonded phase fused silica capillary column.

\section{Solid-state NMR}

Solid-state ${ }^{13} \mathrm{C}$ NMR experiments were carried out in a Bruker Avance III 600 WB with a magnetic field of 17.09 $\mathrm{T}$ and equipped with a $4 \mathrm{~mm}$ multinuclear probe MAS NMR. ${ }^{13} \mathrm{C}$ resonance frequency in this magnetic field is $150.9 \mathrm{MHz}$.

NMR experiments were performed on the untreated sample and also on the solid biomass fractions resulting from enzymatic hydrolysis samples were packed in zirconium oxide rotors and were spun at $12 \mathrm{kHz}$.

The proton decoupled ${ }^{13} \mathrm{C}$ NMR experiments were carried out using a pulse of $30^{\circ}$ of $1.37 \mu$ s for ${ }^{13} \mathrm{C}$, a relaxation time of $10 \mathrm{~s}$, acquisition time of $0.031 \mathrm{~s}$ and a scan number of 4800 , corresponding to $11 \mathrm{~h}$ and $10 \mathrm{~min}$ for each sample. The chemical shifts are expressed in ppm and they were referenced with TMS $(\delta=0 \mathrm{ppm})$.

Assignments of peaks are described in Table 1 based on previous NMR analysis on literature $[12,13]$.

\section{Results and discussion \\ Composition of pretreated corn stover and pretreated sugarcane straw samples}

The compositional analysis of each material was carried out as described in materials and method. Table 2 shows carbohydrates (cellulose and hemicellulose components) and lignin composition of both materials. The insoluble part of each material showed some similarities relating to insoluble sugar content, although pretreated sugarcane straw exhibited more xylan content than pretreated corn stover, probably due to a lower effect of the pretreatment. A small percentage of mannan is present in pretreated sugarcane straw, but is not detected in pretreated corn stover.

\section{Enzymatic hydrolysis of pretreated corn stover and pretreated sugarcane straw}

Samples were hydrolyzed using a cellulolytic cocktail produced by $\mathrm{C} 1$ strain of Myceliophthora thermophila [14]. This strain developed by Abengoa is able to achieve more than $100 \mathrm{~g} / \mathrm{L}$ of total extracellular protein at the industrial-scale fermenters. More than $90 \%$ of the extracellular protein produced consists of a mixture of cellulases, of which $40-55 \%$ are cellobiohydrolases, $20-25 \%$ are endoglucanases among others betaglucosidases, betaxilosidases, polysaccharide monooxygenases, xylanases and xyloglucanases, arabinofuranosidases, acetylxylan esterases, and alfa and betagalactosidases. Due to its lower ethanol cost contribution, this enzymatic cocktail produced is preferred instead of others from wellknown fungi like Aspergillus sp. or Trichoderma reesei for recently developed biorefineries. Figure 1 shows the total sugar yield achieved for the two pretreated substrates in 
Table 1 Assignment of NMR peaks 1-17 indicated on the spectrum in Fig. 3

\begin{tabular}{|c|c|c|}
\hline $\begin{array}{l}\text { Peak } \\
\text { number }\end{array}$ & Chemical group & $\begin{array}{l}{ }^{13} \mathrm{C} \text { chemical } \\
\text { shift (ppm) }\end{array}$ \\
\hline 1 & $\begin{array}{l}\text { Aliphatic lignin carbons not bound to } \\
\text { oxygen }\end{array}$ & 32.5 \\
\hline 2 & Aryl methoxyl carbons of lignin & 56.2 \\
\hline 3 & $\begin{array}{l}\text { C6 carbon of non-crystalline/amorphous } \\
\text { cellulose, } \mathrm{C} 6 \text { carbon of hemicelluloses, } \\
\mathrm{OC} \mathrm{H} 2 \text { carbons of lignin }\end{array}$ & 62.5 \\
\hline 4 & C6 carbon of crystalline cellulose & 64.8 \\
\hline 5 & $\begin{array}{l}\mathrm{C} 2,3,5 \text { of cellulose, } \mathrm{OCaH} 2 \text { carbons of } \\
\text { lignin }\end{array}$ & 72.5 \\
\hline 6 & $\mathrm{C} 2,3,5$ of cellulose and hemicelluloses & 74.4 \\
\hline 7 & $\begin{array}{l}\text { C4 carbon of non-crystalline cellulose } \\
\text { and hemicelluloses, } \mathrm{OC} \beta \mathrm{H} 2 \text { carbons of } \\
\text { lignin }\end{array}$ & 83.5 \\
\hline 8 & C4 carbon of crystalline cellulose & 87.9 \\
\hline 9 & C1 carbon of hemicelluloses & 101.8 \\
\hline 10 & C1 carbon of cellulose & 105.0 \\
\hline 11 & $\begin{array}{l}\mathrm{C} 2 \text { and } \mathrm{C} 6 \text { aromatic carbons of syringyl } \\
\text { and } \mathrm{C} 5 \text { and } \mathrm{C} 6 \text { aromatic carbons of } \\
\text { guaiacyl in lignin }\end{array}$ & $110.0-115.0$ \\
\hline 12 & C2 of aromatic carbons guaiacyl in lignin & 126.6 \\
\hline 13 & $\mathrm{C} 1$ and $\mathrm{C} 4$ aromatic carbons of syringyl ${ }^{\mathrm{a}}$ & 136.9 \\
\hline 14 & $\begin{array}{l}\mathrm{C} 3 \text { and } \mathrm{C} 5 \text { aromatic carbons of syringyl } \\
\text { and } \mathrm{C} 1 \text { and } \mathrm{C} 4 \text { aromatic carbons of } \\
\text { guaiacyl in lignin }\end{array}$ & 148.0 \\
\hline 15 & $\begin{array}{l}C 3 \text { and } C 5 \text { aromatic carbons of syringyl }{ }^{b} \\
\text { in lignin }\end{array}$ & 153.5 \\
\hline 16 & Carboxyl groups of lignin & $163.0-180.3$ \\
\hline 17 & Carboxyl groups of hemicelluloses & 173.6 \\
\hline
\end{tabular}

These assignments were based on published material

a Non-etherified arylglycerol $\beta$-aryl ethers

b Etherified arylglycerol $\beta$-aryl ethers

response to increases in enzyme dose. For both materials, assays were done at $20 \%$ total solids at $50{ }^{\circ} \mathrm{C}$ for $72 \mathrm{~h}$. Above $50 \mathrm{mg}$ of enzyme per gram of glucan, the sugar yield reached $90 \%$ and did not increase further, leaving about $10 \%$ of potential sugar unreleased.

This gap between theoretic potential sugars and real yield at high enzyme loading might be explained by two hypothesis: either pretreatment of the material is not enough to recover all potential sugars present in the biomass, or the structure of the recalcitrant material is complex and new or different accessory enzymes are needed to release all C5 or C6 monomeric sugars. To elucidate this, different approaches were undertaken using glycosyl analysis of the recalcitrant materials.

\section{Glycosyl composition analysis}

Glycosyl composition analysis of the recalcitrant material was performed by combined GC/MS of the
Table 2 Chemical composition of the insoluble pretreated corn stover and pretreated sugarcane straw samples

\begin{tabular}{llc}
\hline $\begin{array}{l}\text { Structural } \\
\text { component }\end{array}$ & \multicolumn{2}{l}{ Insoluble biomass composition (\% DM) } \\
\cline { 2 - 3 } & $\begin{array}{l}\text { Pretreated corn } \\
\text { stover }\end{array}$ & $\begin{array}{l}\text { Pretreated sugarcane } \\
\text { straw }\end{array}$ \\
\hline $\begin{array}{l}\text { Cellulose } \\
\text { Glucan }\end{array}$ & $34.42 \pm 0.21$ & $34.52 \pm 0.32$ \\
Hemicellulose & $3.26 \pm 0.36$ & $4.37 \pm 0.40$ \\
Xylan & $0.33 \pm 0.04$ & $0.55 \pm 0.07$ \\
Arabinan & $0.00 \pm 0.00$ & $0.24 \pm 0.03$ \\
Mannan & $18.03 \pm 0.08$ & $18.51 \pm 0.05$ \\
Klason lignin & $2.40 \pm 0.10$ & $2.41 \pm 0.08$ \\
Ash & $58.44 \pm 0.79$ & $60.59 \pm 0.95$ \\
Total &
\end{tabular}

Contents are expressed on a dry weight basis as an average ( \pm standard deviation) of duplicate determinations

Samples were pretreated using the two-stage acid hydrolysis method as described in experimental procedures

per-O-trimethylsilyl derivatives of the monosaccharide methyl glycosides produced from the samples by acidic methanolysis. The data are presented in Table 3.

Only five different monosaccharides were detected using this analytical technique (arabinose, glucose, xylose, mannose and galactose), and those monomers that were more represented in both materials were glucose and xylose, matching with the previous compositional analysis (Table 2). No other saccharides such as ribose, rhamnose, fucose, glucuronic acid, galacturonic acid, $\mathrm{N}$-acetyl galactosamine, $\mathrm{N}$-acetyl glucosamine, and $\mathrm{N}$-acetyl mannosamine were detected in any material. Galactose was only detected in the starting materials at low concentration, but not in the insoluble portion after enzymatic hydrolysis. As expected, at higher enzyme dosage, the glucose content present in recalcitrant

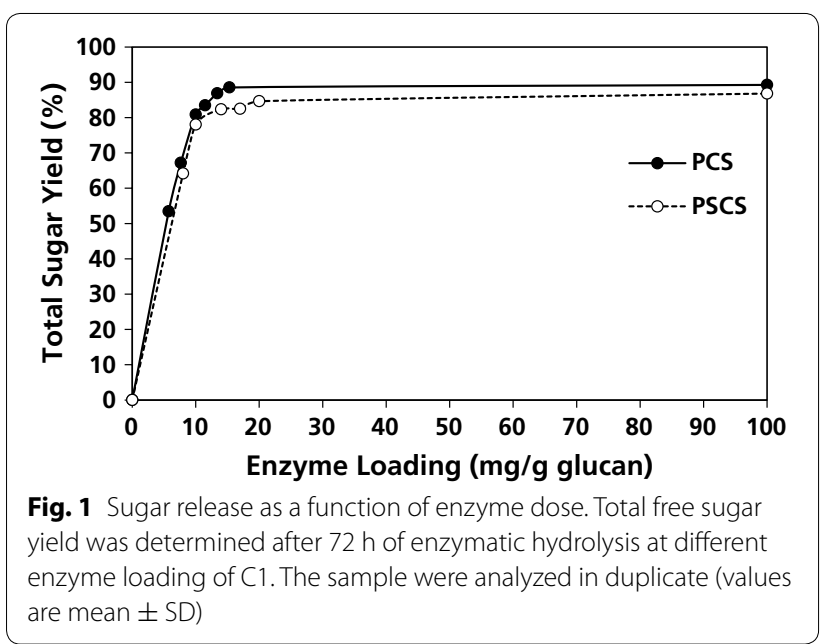


Table 3 Glycosyl residue content

\begin{tabular}{|c|c|c|c|c|c|c|}
\hline \multirow[t]{3}{*}{ Dosage $(\mathrm{mg} / \mathrm{g})$} & \multicolumn{6}{|c|}{ Glycosyl residue content (mol \%; Min-max) } \\
\hline & \multicolumn{3}{|l|}{ PCS } & \multicolumn{3}{|l|}{ PSCS } \\
\hline & 0 & 10 & 100 & 0 & 10 & 100 \\
\hline \multicolumn{7}{|l|}{ Residue } \\
\hline Glucose & $31.7-33.3$ & $32.4-36.1$ & $26.4-26.6$ & $16.9-18.2$ & $38.7-40.7$ & $28.6-30.6$ \\
\hline Xylose & $51.1-53.4$ & $51.1-53.4$ & $53.6-54.8$ & $64.8-66.9$ & $45.4-47.0$ & $46.5-50.7$ \\
\hline Arabinose & $13.0-13.1$ & $11.6-13.2$ & $15.8-16.6$ & $14.8-15.4$ & $12.1-12.4$ & $14.0-14.7$ \\
\hline Mannose & $0.5-0.7$ & $1.0-1.2$ & $2.9-3.4$ & $0.8-1.0$ & $1.8-1.9$ & $6.7-8.1$ \\
\hline Galactose & $1.3-1.9$ & nd & nd & $0.6-0.7$ & nd- -0.4 & nd \\
\hline Total monosaccharides (mg/g) & $221.6-301.1$ & $37.1-71.6$ & $21.4-31.4$ & $250.9-653.5$ & $51.4-51.8$ & $25.7-39.4$ \\
\hline
\end{tabular}

Ribose, rhamnose, fucose, glucuronic acid, galacturonic acid, $\mathrm{N}$-acetyl galactosamine, $\mathrm{N}$-acetyl glucosamine and $\mathrm{N}$-acetyl mannosamine were not detected in any material

Glycosyl residue content ( $\mathrm{mol} \%$ ) of each sample at initial time (dosage $0 \mathrm{mg} / \mathrm{g}$ ), and the insoluble part after enzymatic hydrolysis at lower dosage (10 mg/g) and higher dosage $(100 \mathrm{mg} / \mathrm{g})$ loading of $\mathrm{C} 1$ enzyme related to total dry biomass

nd not detected

material as cellulose and hemicellulose diminished compared to low dosages (Table 3). Comparing different enzyme dosages with non-hydrolyzed material monosaccharides proportions present in the recalcitrant material of corn stover remains almost constant. PSCS shows the highest xylose percentage, but it is reduced enzymatically to similar percentages than obtained with PCS. Mannose level was almost constant at low dosage; however, at the high dosage, this sugar became more abundant in both recalcitrant materials. This fact might indicate that mannose links in the recalcitrant material are not released by the enzymatic cocktail. To identify the different chemical bonds linking all these carbohydrates, a glycosyl linkage analysis was performed.

For glycosyl linkage analysis, per- $O$-methylation and linkage analysis of neutral sugars was carried out. The sample was permethylated, depolymerized, reduced, and acetylated; and the resulting PMAAs analyzed by GC/ MS. Linkage types detected using this technique were those corresponding to the five monosaccharides formerly determined by TMS analysis, showing no other sugars in the biomass composition (Table 4). A schematic diagram of each glycosyl linkage type is shown in the Fig. 2.

According to per-O-methylation and linkage analysis, 4 linked glucopyranosyl residue is the major component of all samples and a significant amount of 4 linked xylopyranosyl residues is also present in all samples. The possible origin of the linked residues detected is presented in Table 5.

The linkage information is based on published identification with model compounds [15]. Apart from the predominant 4 linked glucopyranosyl residues mentioned, four additional types of glucose linkages were detected.
Among them, 6-linked glucopyranosyl residues that were not detected in the initial material were present in a small proportion after enzymatic hydrolysis regardless of the dosages. This residue might be released by the action of different enzymes, like, depending on the modifications present, acetylxylan-feruloyl esterases or $\alpha$-arabinofuranosidases.

The 3-linked glucopyranosyl residues became less represented after enzymatic hydrolysis compared to the initial material and remained almost constant at lower and higher enzyme loading.

In the case of xylose residues, 3,4-linked xylopyranosyl residues increased their proportion after hydrolysis with higher loading on the enzyme. These kinds of links are usually found in arabinoxylan structures; consequently, enzymes that could be involved would be arabinofuranosidase, xylanase or beta-xylosidase, among others.

On the other hand, arabinopyranosyl, galactopyranosyl, and mannopyranosyl residues remained more or less constant in all conditions and were less abundant.

It has to be noted that quantitative composition analysis data are inconsistent with the linkage analysis data. The linkage analysis indicated higher amounts of 4-linked glucopyranosyl residues in all samples, while composition analysis suggested lesser amount of glucose. These results can be explained by the fact that the permethylation step in linkage analysis allowed to solubilize cellulose resulting in more efficient hydrolysis.

All these analyses revealed that most linkages belonged to glucan and xylan, specifically, beta-1,4-glucan and beta1,4-xylan. Therefore, although there are some residues from other carbohydrates, the main recalcitrant material would be composed of these two polymers. Enzymes that are involved in their breakdown are endoglucanases, 
Table 4 Glycosyl linkage content

\begin{tabular}{|c|c|c|c|c|c|c|c|}
\hline \multirow[t]{3}{*}{ Residue linkage type } & \multirow[t]{3}{*}{ Dosage (mg/g) } & \multicolumn{6}{|c|}{ Glycosyl linkage content (\%) } \\
\hline & & \multicolumn{3}{|l|}{ PCS } & \multicolumn{3}{|c|}{ PSCS } \\
\hline & & 0 & 10 & 100 & 0 & 10 & 100 \\
\hline \multirow[t]{5}{*}{ Glucopyranosyl } & Terminally linked (1) & 5.4 & 3.5 & 6.2 & 7.2 & 9.4 & 7.1 \\
\hline & 3 linked (2) & 1.9 & 0.4 & 0.5 & 1.2 & 0.5 & 0.6 \\
\hline & 4 linked (3) & 63.3 & 72.1 & 62.8 & 71.6 & 67.8 & 52.7 \\
\hline & 6 linked (4) & 0.0 & 0.3 & 0.4 & 0.0 & 0.5 & 0.9 \\
\hline & 4,6 linked (5) & 1.6 & 1.8 & 1.7 & 1.5 & 1.8 & 2.3 \\
\hline \multirow[t]{3}{*}{ Xylopyranosyl } & 4 linked (6) & 13.6 & 11.3 & 13.4 & 8.0 & 8.7 & 13.4 \\
\hline & 2,4 linked (7) & 0.0 & 0.4 & 0.3 & 0.0 & 0.3 & 0.0 \\
\hline & 3,4 linked (8) & 0.9 & 1.3 & 2.0 & 0 & 1.3 & 3.6 \\
\hline \multirow[t]{3}{*}{ Arabino(pyra/fyra)nosyl (Arap/Araf) } & Terminally linked (9) & 4.8 & 1.2 & 1.8 & 2.8 & 1.6 & 2.9 \\
\hline & Terminally linked (10) & 0.9 & 2.0 & 1.7 & 0.4 & 1.7 & 5.3 \\
\hline & 4 linked Arap or 5 linked Araf $(11)^{a}$ & 1.0 & 0.0 & 1.9 & 0.0 & 1.9 & 5.6 \\
\hline \multirow[t]{4}{*}{ Mannopyranosyl } & Terminally linked (12) & 0.7 & 0.1 & 1.1 & 0.7 & 0.6 & 1.4 \\
\hline & 2 linked (13) & 0.0 & 0.0 & 0.7 & 0.0 & 0.0 & 0.9 \\
\hline & 4 linked (14) & 2.7 & 1.1 & 0.5 & 3.4 & 1.0 & 0.0 \\
\hline & 6 linked (15) & 0.0 & 0.0 & 0.1 & 0.0 & 0.0 & 0.0 \\
\hline \multirow[t]{3}{*}{ Galactopyranosyl } & Terminally linked (16) & 0.9 & 1.0 & 0.6 & 0.9 & 0.3 & 0.0 \\
\hline & 2,4 linked (17) & 0.6 & 0.8 & 1.7 & 0.6 & 0.8 & 0.5 \\
\hline & 3,4 linked (18) & 1.4 & 1.7 & 1.6 & 1.4 & 1.1 & 1.7 \\
\hline
\end{tabular}

a 4-linked Arap and 5-linked Araf give rise to the same PMAA and can thus not be distinguished by this method. Numbers within parentheses are related with the drawings in Fig. 2

Glycosyl linkage residue of each sample at initial time (dosage $0 \mathrm{mg} / \mathrm{g}$ ), and the insoluble part after enzymatic hydrolysis at lower dosage (10 mg/g) and higher dosage $(100 \mathrm{mg} / \mathrm{g})$ loading of $\mathrm{C} 1$ enzyme related to total dry biomass. Samples were performed in duplicate

cellobiohydrolases, and beta-glucosidases in the case of glucan, and xylanase and beta-xylosidase, in the case of xylan, enzymes that are already present in the $\mathrm{C} 1$ enzymatic cocktail preparation. The same results were also obtained repeating the same analysis with other available commercial cellulolytic cocktail like Ctec3 from Novozymes (data not shown).

Because the enzymes required to hydrolyze the types of linkages found are abundant in the enzyme cocktails used and, moreover, they have been able to solubilize most of the material in the prehydrolysis phase, we could deduce that the recalcitrant material remaining is not accessible to the enzymes and, hence, it cannot be hydrolyzed. These pockets of inaccessible recalcitrant polysaccharides could come from an incomplete pretreatment of the starting biomass.

\section{Solid-state ${ }^{13} \mathrm{C}$ nuclear magnetic resonance}

Further information on chemical composition for recalcitrant samples was obtained by high-resolution ${ }^{13} \mathrm{C}$ solid-state nuclear magnetic resonance. For organic matter applications, one of the most quantitative ${ }^{13} \mathrm{C}$ NMR techniques is probably the DPMAS method, consisting of the excitation of the ${ }^{13} \mathrm{C}$ nuclei by a single $\pi / 2$ pulse, followed by acquisition under ${ }^{1} \mathrm{H}$ decoupling and fast magic-angle spinning. This method can readily provide information on modifications taking place in specific chemical groups, without great experimental effort concerning sample preparation and analysis, which makes it fast $[16,17]$.

Figure 3 shows the ${ }^{13} \mathrm{C}$ NMR spectrum for the PCS samples at different enzyme loadings. This spectrum is very similar to those described in the literature [18-20].

The cellulose region $(60-110 \mathrm{ppm})$ is typical of pretreated corn stover samples. Signals 3 and 7 (at 63 and $84 \mathrm{ppm}$ ) were assigned, respectively, to $\mathrm{C} 6$ and $\mathrm{C} 4$ carbon from amorphous cellulose. On the other hand, signal 4 and 8 (at 65 and $88 \mathrm{ppm}$ ) were assigned to C6 and C4 carbon in crystalline cellulose. It is difficult to clearly differentiate the signals of lignin, hemicellulose, and cellulose because there is a contribution of all bound carbons along the entire spectral region. However, contribution of hemicellulose and lignin is smaller in the region from 60 to $110 \mathrm{ppm}$. Lignin signal becomes more important from 110 to $180 \mathrm{ppm}$, while hemicellulose signals contribute to peaks $3,6,7,8,9$, and 17 .

Comparing the spectra at the two enzyme loadings, the same peaks from cellulose signals $(3,4,5,6,7,8$, and 10) 

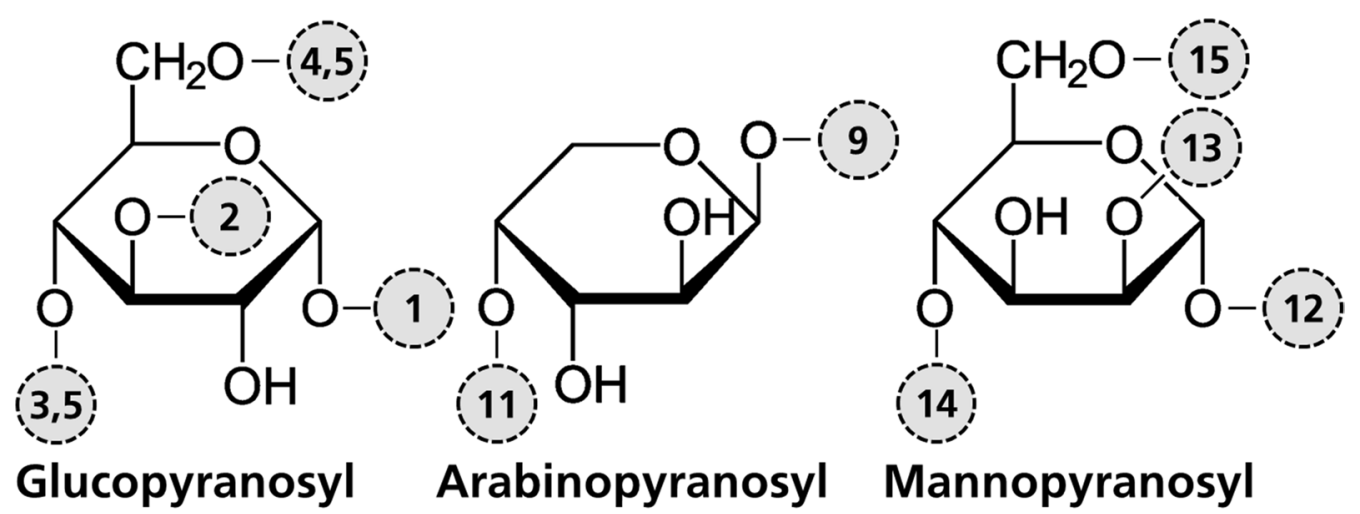

\section{Glucopyranosyl}

\section{Mannopyranosyl}
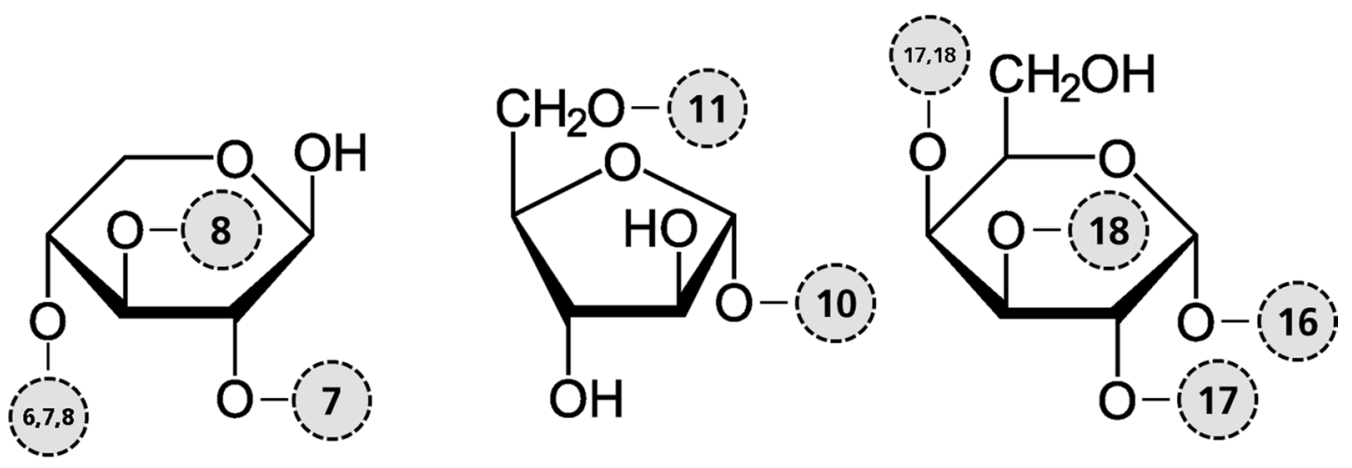

\section{Xylopyranosyl}

\section{Arabinofuranosyl}

\section{Galactopyranosyl}

Fig. 2 Schematic structure of linkage type on model compounds. Glucopyranosyl residues: terminally linked (1), 3 linked (2), 4 linked (3), 6 linked (4), 4,6 linked (5); Xylopyranosyl residues: 4 linked (6), 2,4 linked (7), 3,4 linked (8); arabino(pyra/fura) nosyl residues: terminally linked pyranosyl (9), terminally linked furanosyl (10), 4 linked pyranosyl or 5 linked furanosyl (11); mannopyranosyl residues: terminally linked (12), 2 linked (13), 4 linked (14), 6 linked (15); galactopyranosyl residues: terminally linked (16), 2,4 linked (17), 3,4 linked (18)

Table 5 Possible origin of the detected linked residues

\begin{tabular}{|c|c|c|c|}
\hline Residue & Linkage type & Model compound $^{\mathrm{a}}$ & Putative enzyme involved \\
\hline \multirow[t]{5}{*}{ Glucopyranosyl } & Terminally linked (1) & Pullulan & $\mathrm{Eg} / \mathrm{Cbh} / \mathrm{Bgl}$ \\
\hline & 3 linked (2) & Lichenan & Ace/Fe \\
\hline & 4 linked (3) & Glucan & $\mathrm{Eg} / \mathrm{Cbh} / \mathrm{Bgl}$ \\
\hline & 6 linked (4) & Pullulan, stachyose & Ace/Fe/Aabn \\
\hline & 4,6 linked (5) & Xyloglucan & $\mathrm{Eg} / \mathrm{Cbh} / \mathrm{Bgl}$ \\
\hline \multirow[t]{2}{*}{ Xylopyranosyl } & 4 linked (6) & Xylan & $\mathrm{Xyl} / \mathrm{Bxl}$ \\
\hline & 2,4 linked and 3,4 linked $(7,8)$ & Arabinoxylan & $\mathrm{Abn} / \mathrm{Xyl} / \mathrm{Bx}$ \\
\hline \multirow[t]{2}{*}{ Arabino(pyra/fyra)nosyl (Arap/Araf) } & Terminally linked $(9,10)$ & Arabinose & Abn \\
\hline & 4-linked Arap or 5-linked Araf (11) & Debranched arabinan & Aabn \\
\hline \multirow[t]{3}{*}{ Mannopyranosyl } & Terminally linked (12) & Mannose & Bman \\
\hline & 2 linked and 6 linked $(13,15)$ & Acetylation $^{\mathrm{b}}$ & Ace/Fe \\
\hline & 4 linked (14) & Mannan & Bman \\
\hline \multirow[t]{2}{*}{ Galactopyranosyl } & Terminally linked (16) & Galactose & Bgal \\
\hline & 2,4 linked and 3,4 linked $(17,18)$ & Galactan ${ }^{b}$ & AGal/Bgal \\
\hline
\end{tabular}

a Based on previously published data

b Proposed hypothetical model compound

Numbers within parentheses are related to drawings in Fig. 2

Abn arabinofuranosidases, Aabn alfaarabinofuranosidases, $X y l$ xylanases, Bxl betaxylosidases, Eg endoglucanases, Cbh cellobiohydrolases, Bg/ betaglucosidases, $A c e$ acetylxylan esterases, Fe feruroyl esterases, Bman betamannosidases, Agal alfagalactosidases, Bgal betagalactosidases

The linkage information is deduced from the knowledge of model compounds 
were observed, indicating that there was still a fraction of cellulose present after enzymatic hydrolysis at the higher enzyme loading (Fig. 3). This would support the hypothesis obtained with the glycosyl analysis about the presence of cellulose and hemicellulose rests blocked to enzyme action. The same results were observed for both PCS and PSCS materials.

\section{Conclusions}

The aim of the present study was to guide the enzymatic cocktail improvement to reduce the recalcitrant carbohydrate fraction characterizing the chemical composition of this material remaining after forced enzymatic hydrolysis of two pretreated lignocellulosic substrates (pretreated corn stover and pretreated sugarcane straw). The analysis was performed using techniques such as NMR or glycosyl residue composition by methylation analysis.

Recalcitrant material in both substrates represented approximately a $10 \%$ of total carbohydrate left without being hydrolyzed. Two hypothesis were proposed to explain this gap: either the necessity of accessory enzymes to release a part of the polymer structure that was blocked by other modifications (such as acetyl/

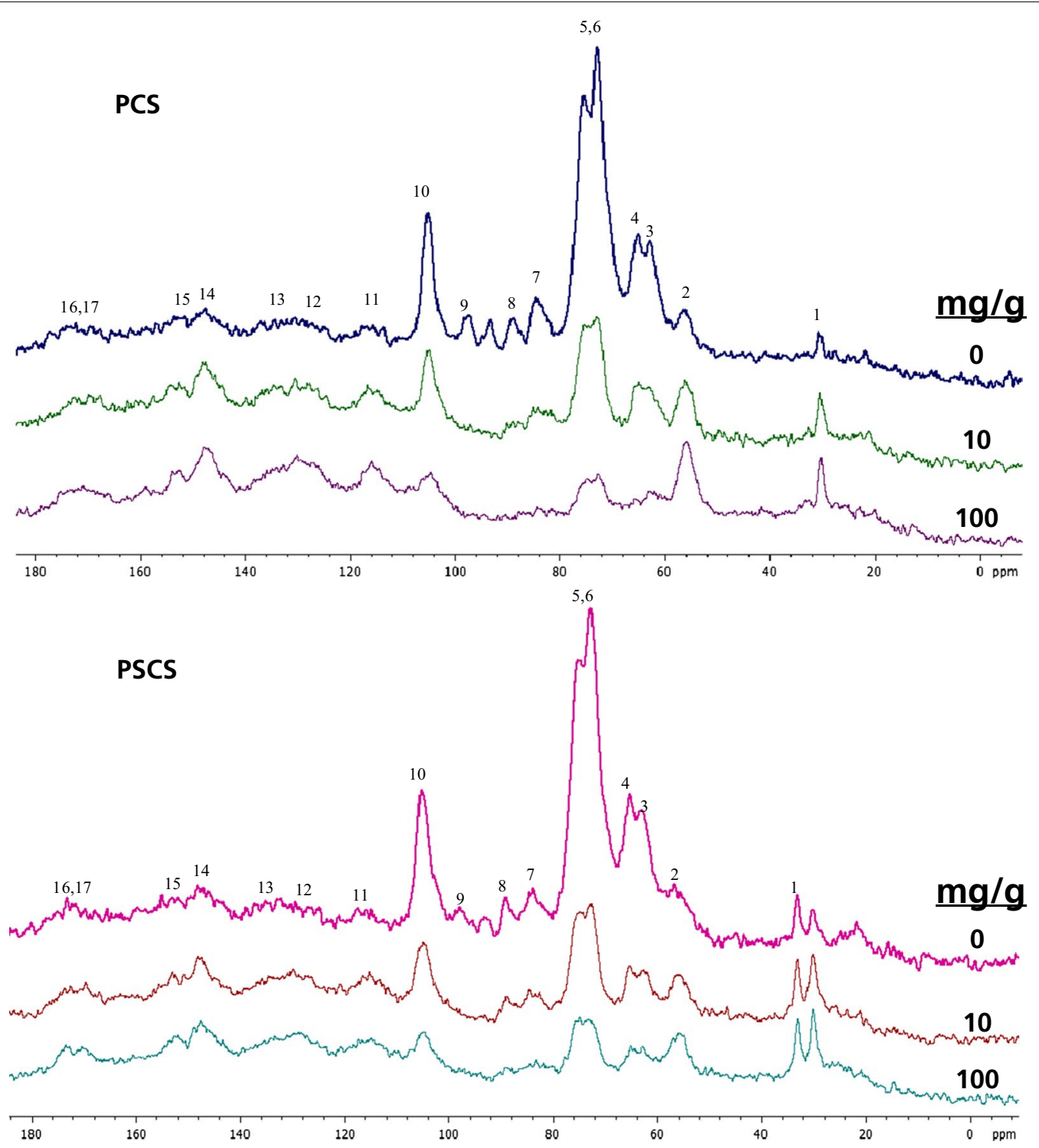

Fig. 3 Identification of 13 signals in non-hydrolyzed and hydrolyzed materials. Solid stage ${ }^{13} \mathrm{C}$ high-power proton decoupling magic-angle spinning $\left({ }^{13} \mathrm{C}\right.$ HPDC/MAS) nuclear magnetic resonance (NMR) spectra of pretreated corn stover (up; PCS) and pretreated sugarcane straw (down; PSCS) at several enzyme loadings before enzymatic hydrolysis ( 0 mg protein/g glucan) and after enzymatic hydrolysis (using 10 and $100 \mathrm{mg}$ protein/g glucan) 
feruloyl resides), or an incomplete pretreatment of the biomass (i.e., due to the irregular particle size distribution or an inefficiently acid-exploded material attributable to high-scale operations), limiting the accessibility of the enzymes to the main polysaccharides of glucan and xylan.

Glycosyl composition analysis reveals that the recalcitrant material remains with glucose and xylose as the main monomers matching with the non-hydrolyzed material. Despite that some links could became slightly more represented, glycosyl linkage analysis reveals that 4-linked glucopyranosyl residue is the major component of all samples followed by a significant amount of 4-linked xylopyranosyl residues.

The results are consistent with the hypothesis that assigns the yield gap in hydrolysis to incomplete pretreatment leading to "pockets" of enzyme-inaccessible materials, instead to the need of alternative enzymes to process specific bonds linking the sugar polymers, because these links or polymers could not be found in the recalcitrant material. Besides according to Solid-state ${ }^{13} \mathrm{C}$ nuclear magnetic resonance studies, this material has shown instead the presence of cellulose and hemicellulose and the chemical links corresponding to them.

This means that the hydrolytic yield limitations of the materials tested are likely caused by an incomplete pretreatment of the biomass and not in the enzyme cocktail preparations, which, on the other hand include all the enzymes required to hydrolyze the polysaccharides (mainly glucan and xylan) present in the recalcitrant material.

\begin{abstract}
Abbreviations
DMSO: dimethyl sulfoxide; DPMAS: direct polarization magic-angle spinning; EI-MS: electron impact ionization mode; GC/MS: gas chromatography/mass spectrometry; HPDC/MAS: high-power proton decoupling magic-angle spinning; HPLC: high-performance liquid chromatography; NMR: nuclear magnetic resonance spectroscopy; NREL: National Renewable Energy Laboratory; PCS: pretreated corn stover; PMAAs: partially methylated alditol acetates; PSCS: pretreated sugarcane straw; TFA: trifluoroacetic acid; TMS: per-O-trimethylsilyl.
\end{abstract}

\section{Authors' contributions}

MABA carried out the enzymatic hydrolysis experiments, participated in the NMR experiments, and drafted the manuscript. JD and PA carried out the glycosyl composition and linkage analysis. FPMH participated in the NMR analysis and helped to draft the manuscript. BD participated in the biomass pretreatment, design of the study and manuscript revision. FMRS conceived the study and participated in the hydrolysis experiments, manuscript revision, and coordination between groups. All authors read and approved the final manuscript.

\section{Author details}

1 Department of Biotechnology, Abengoa Research, Campus Palmas Altas, C/ Energía Solar no 1, 41014 Seville, Spain. ${ }^{2}$ Complex Carbohydrate Research Center, University of Georgia, Athens 30602, Georgia. ${ }^{3}$ Instituto de Bioquímica Vegetal y Fotosíntesis, Universidad de Sevilla y CSIC, Américo Vespucio 49, 41092 Seville, Spain.

\section{Acknowledgements}

Part of this work was supported by grants from the Chemical Sciences, Geosciences and Biosciences Division, Office of Basic Energy Sciences, US Department of Energy grant (DE-FG02-93ER20097) to Parastoo Azadi at the Complex Carbohydrate Research Center. Work in Abengoa was supported by grants of the European Commission within FP7-NMP (Grant agreement No: 604530) and the Spanish MINECO (PCIN-2013-012-C02-02). The authors thank Juan Luis Ramos for critical reading of the manuscript.

\section{Competing interests}

The authors declare that they have no competing interests.

Received: 17 June 2016 Accepted: 28 September 2016

Published online: 06 October 2016

\section{References}

1. Wyman CE. Biomass ethanol: technical progress, opportunities, and commercial challenges. Annu Rev Energy Environ. 1999;24:189-226.

2. Chandra RP, Bura R, Mabee WE, Berlin A, Pan X, Saddler JN. Substrate pretreatment: the key to effective enzymatic hydrolysis of lignocellulosics? Adv Biochem Eng Biotechnol. 2007;108:67-93.

3. Agbor VB, Cicek N, Sparling R, Berlin A, Levin DB. Biomass pretreatment: fundamentals toward application. Biotechnol Adv. 2011;29:675-85.

4. Foston M, Ragauskas AJ. Biomass characterization: recent progress in understanding biomass recalcitrance. Ind Biotechnol. 2012;8:191-208.

5. Sweeney MD, Xu F. Biomass converting enzymes as industrial biocatalysts for fuels and chemicals: recent developments. Catalysts. 2012;2:244-63.

6. Sun Y, Cheng J. Hydrolysis of lignocellulosic materials for ethanol production: a review. Bioresour Technol. 2002:83:1-11.

7. Biomass compositional analysis laboratory procedures. National Renewable Energy Laboratory (NREL). 2016. http://www.nrel.gov/bioenergy/ biomass-compositional-analysis.html. Accessed 06 Jun 2016.

8. Kristensen JB, Felby C, Jørgensen $\mathrm{H}$. Determining yields in high solids enzymatic hydrolysis of biomass. Appl Biochem Biotechnol. 2009;156:127-32.

9. Merkle RK, Poppe I. Carbohydrate composition analysis of glycoconjugates by gas-liquid chromatography/mass spectrometry. Methods Enzymol. 1994;230:1-15 (American Chemical Society).

10. York WS, Darvill AG, McNeil M, Stevenson TT, Albersheim P. Isolation and characterization of plant cell walls and cell wall components. Methods Enzymol. 1986;1 18:3-40 (American Chemical Society)

11. Ciucanu I, Kerek F. A simple and rapid method for the permethylation of carbohydrates. Carbohydr Res. 1984;131:209-17.

12. Liitia T, Maunu SL, Hortling B. Solid-state NMR studies of residual lignin and its association with carbohydrates. J Pulp Pap Sci. 2000;26:323-30.

13. Ciesielski PN, Wang W, Chen X, Vinzant TB, Tucker MP, Decker SR, Himmel $\mathrm{ME}$, Johnson DK, Donohoe BS. Effect of mechanical disruption on the effectiveness of three reactors used for dilute acid pretreatment of corn stover part 2: morphological and structural substrate analysis. Biotechnol Biofuels. 2014;7:47.

14. Visser H, Joosten V, Punt PJ, Gusakov AV, Olson PT, Joosten R, Bartels J, Visser J, Sinitsyn AP, Emalfarb MA, Verdoes JC, Wery J. Development of a mature fungal technology and production platform for industrial enzymes based on a Myceliophthora thermophila isolate, previously known as Chrysosporium lucknowense C1. Ind Biotechnol. 2011;7:214-24.

15. Laine C, Tamminen T, Vikkula A, Vuorinen T. Methylation analysis as a tool for structural analysis of wood polysaccharides. Holzforschung. 2002;56:607-14.

16. Cook RL. Coupling NMR to NOM. Anal Bioanal Chem. 2004;378:1484-503.

17. Smernik RJ, Olk DC, Mahieu N. Quantitative solid-state ${ }^{13}$ C NMR spectroscopy of organic matter fractions in lowland rice soils. Eur J Soil Sci. 2004:55:367-79.

18. Rezende C, de Lima M, Maziero P, de Azevedo E, Garcia W, Polikarpov I. Chemical and morphological characterization of sugarcane bagasse submitted to a delignification process for enhanced enzymatic digestibility. Biotechnol Biofuels. 2011:4:54 
19. Walker TE, London RE, Whaley TW, Barker R, Matwiyoff NA. Carbon-13 nuclear magnetic resonance spectroscopy of $\left[{ }^{1-13} \mathrm{C}\right]$ enriched monosac charides. Signal assignments and orientational dependence of geminal and vicinal carbon-carbon and carbon-hydrogen spin-spin coupling constants. J Am Chem Soc. 1976;98:5807-13.
20. Wikberg H, Maunu SL. Characterisation of thermally modified hard- and softwoods by ${ }^{13} \mathrm{C}$ CPMAS NMR. Carbohydr Polym. 2004;58:461-6.
Submit your next manuscript to BioMed Central and we will help you at every step:

- We accept pre-submission inquiries

- Our selector tool helps you to find the most relevant journal

- We provide round the clock customer support

- Convenient online submission

- Thorough peer review

- Inclusion in PubMed and all major indexing services

- Maximum visibility for your research

Submit your manuscript at www.biomedcentral com/submit
() Biomed Central 